\title{
Rüb, Friedbert W. (2020): Das Jahrhundert der Politik. Eine Geschichte des 20. Jahrhunderts im Licht ihrer Politikbegriffe
}

\author{
Baden-Baden: Nomos. 682 Seiten. $138 €$
}

\author{
Sebastian Huhnholz
}

Angenommen: 22. Dezember 2020 / Online publiziert: 7. Januar 2021

(C) Der/die Autor(en) 2021

Das enorme Spektrum des hier anzuzeigenden Buchs von Friedbert W. Rüb einzufangen, ist unmöglich. Allein eine Übersicht würde den Rahmen sprengen. Bloße Schlaglichter drohten, den Anspruch zu unterschlagen. Das Buch will eine „Geschichte des 20. Jahrhunderts [...] durch die Brille von relevanten politischen Semantiken“ entwickeln (S. 25). Es steigt ein mit Reflexionen zur neueren Mode, „Politik“ und das „Politische“ zu unterscheiden, betrachtet die „Politik der Verfassungsgebung " zwischen Oktoberrevolution und den osteuropäischen Transformationen, widmet sich Theorien der Masse (von Gustave Le Bon über Sigmund Freud und Wilhelm Reich bis René König sowie Michael Hardt \& Antonio Negri), dem Wohlfahrtsstaat, der „Politik des Tötens“, des „Krieges“, des „Friedens“ und vielem anderen mehr.

Abkürzend sei auf die Titelgebung verwiesen: „Das Jahrhundert der Politik“ im Lichte ihrer nicht auf „Semantik“ reduzierbaren Begrifflichkeiten nämlich impliziert sowohl, dass das letzte Jahrhundert sich durch eine besondere Aufmerksamkeit für das Wesen der Politik auszeichnete wie auch, dass die dabei erreichte, bisweilen monströse Reichweite und Eingriffstiefe vorbei, das Primat der Politik erledigt sei. Folgerichtig handelt das finale Kapitel vom „Verschwinden“ der Politik. Neben das bloß übliche, ,triviale Verschwinden“ in den Hinterzimmern trete ein ,komplexes“: das Verschwinden der großen „Projekte“ und „,der staatlichen bzw. politischen Akteure“ zugunsten eines ,situativen Regieren[s] ohne weitreichenden Gestaltungsanspruch“ (S. 626-635).

An den Beginn dieses Bogens stellt Rüb den ,bahnbrechende[n] Text“ (S. 63) Albert Schäffles von 1897: „Über den wissenschaftlichen Begriff der Politik“. Schäffle, einer der Gründerväter der Soziologie, aber in vielen Disziplinen beheimateter Au-

S. Huhnholz ( $\bowtie)$

Institut für Politische Wissenschaft, Leibniz-Universität Hannover, Hannover, Deutschland

E-Mail: s.huhnholz@ipw.uni-hannover.de 
todidakt, ist von der Rezeption belächelt worden. Ein Klassiker ist er mitnichten. Doch habe er ,den Politikbegriff von allen feststehenden und vorgegebenen Prämissen gelöst [...] und zu einem Prozessbegriff umgeschmolzen“, der den „Status Quo dauerhaft in Frage stellt, neue Optionen und Möglichkeiten kreiert - und diesen Möglichkeitshorizont durch verbindliche Entscheidungen vorübergehend schließt" (S. 626). Wer hier Michael Th. Grevens These der „Politischen Gesellschaft“ anklingen hört, hört richtig: Moderne Politik gilt auch Rüb als Charakter eines ,neuen Gesellschaftstyp[s]“" (S. 23), als Erfassung, Gestaltung und Lenkung potenziell aller Lebensbereiche im Bewusstsein prinzipieller Kontingenz. Just darum muss sie gebändigt und gegen Ewigkeitswahn geschützt werden. Just darum aber auch ist sie mehr als Staat, Überzeugung oder Programm.

So zeigt Rüb, wie stark Schäffle die späteren Diskurse um den Politikbegriff prägte. Die Entwicklung des Politik- als Handlungsbegriff, der nicht mehr auf den Staat begrenzt ist, sieht Rüb bei Schäffle angelegt und zu Karl Mannheim, Max Weber (Bretter „,bohren“, Macht „erstreben“ usf.) und sicher auch Carl Schmitt wandern. Reinhart Kosellecks These der Aneignung, Dynamisierung und Ideologisierung moderner Leitsemantiken klingt hier an; der Berufsrevolutionär wird denkbar. In diesem Sinne also kann auch Politik „Beruf“ werden: Sie ist Tätigkeit, nicht bloß System, Konvention, Institution oder Governance. Und nebenbei sieht Rüb wohl auch richtig, dass die zu oft auf Klassikerpflege trainierte Ideengeschichte ihre Aufgabe verfehlt, wenn sie bloß auf Epigonen wie Weber oder überhaupt auf die Theoriebildungsgeschichte starrt, die Tätigkeit, das Machen von Politik durch Menschen, „Politikertypen“, auch durch Staatsmann und „Staatsfrau“ (S. 585), indes abblendet.

Die lange Vorgeschichte sowohl der deutschsprachigen wie der klassischen und internationalen Semantiken der Politik klammert Rüb aus. Auch über den Bruch, den Weber und Schäffle versuchen, wenn sie sich von Heinrich von Treitschkes berüchtigter „Politik“-Vorlesung absetzen, erfährt man nichts. Dafür werden Theorie-, Fach- und Politikgeschichte ineinander verwoben. Damit übt Rüb Distanz zur Schriftgläubigkeit seiner textlastigen Disziplin, denn auch performative und mediengeschichtliche (z. B. die „Politik mit dem Bild“, der fotografierende Frontsoldat usw., S. 601) Innovationen der neuzeitlichen Politik geraten so in den Blick.

Dass das längste Kapitel moderner Wohlfahrtsstaatlichkeit gewidmet ist, versteht sich. Rüb, Nachfolger Claus Offes auf dem Lehrstuhl für Politische Soziologie und Sozialpolitik der Humboldt-Universität, weiß sich in die Tradition einer politisch bewussten Generation von Sozial- und PolitiktheoretikerInnen zu stellen. Ein echter Gewinn etwa ist Rübs (Wieder-)Eingemeindung Thomas H. Marshalls in den Kanon. Die Idee der Statusgleichheit von Personen durch sozialrechtlich unterfangene Staatsbürgerschaftsrechte hatte kurzzeitig die herablassende Klassifizierung und Disqualifizierung ökonomisch Abhängiger und Bedürftiger dementiert. Hier hatte das „Jahrhundert der Politik“ einen großartigen normativen Referenzpunkt erlangt, der „sich nicht vergessen“ (S. 242) lässt, obwohl wir ihn preisgegeben haben.

Müsste es an nun an dieser breiten Darstellung und beeindruckenden Sammlung überhaupt Kritik geben, könnte sie drei Punkte umfassen. Erstens interessiert den Autoren die Forschung nicht. Kaum ein Kapitel greift auf Fachliteratur zurück, die jünger als 20 Jahre ist. Oft ist sie um ein sehr vieles älter. Den Forschungsstand braucht es sicher nicht immer. Manchmal aber, etwa mit Blick auf die soziale Politik- 
seite des Nationalsozialismus (S. 228-233), verblüfft dieser Mut zur Nachlässigkeit. Auch gemessen daran, dass Rüb für die oft knappe Wiedergabe von KlassikerInnen ganz seinen eigenen Leseeindrücken und Urteilen seines engeren kollegialen Umfelds vertraut, hätte, zweitens, etwas mehr Kontextualisierung, Philologie und Hermeneutik, im Übrigen auch wirkliche Semantologie, der Präzision durchaus gedient. Über die Netzwerke und Politikbedingungen beispielsweise, in denen Schäffle und Weber ihr Denken entfalten, sind ganze Akademieprojekte entstanden. Die Gegenüberstellung eines einzigen Textes von Schäffle hier, Weber da, lässt das beiseite. Sogar naheliegende Querverbindungen unterhalb der Kapitel, etwa zu Robert Michels Eliten-, Parteien- und Massensoziologie, bleiben Andeutungen. So entsteht ein gewisser Vorlesungscharakter des dafür gut lesbaren, äußerst verständlich geschriebenen Buchs.

Und schließlich noch die Frage, ob die Diagnose eigentlich stimmt. Die ,große Politik“ mag vorbei sein und dem Durchwurschteln weichen, dem „Fahren auf Sicht“", der büro- und technokratischen Herrschaft. Das ist hier nicht zu entscheiden, allemal aber diskussionswürdig. Interessanterweise erinnert das an Webers Klage vor 100 Jahren, am Ende des kaiserzeitlichen Ancien Régime, mit dem Rüb auch einsetzt. Weber freilich hatte keinen Mangel an großen Charakteren verspürt, sondern Feigheit eines saturierten Bürgertums, die „Politik“ zu erobern. Mit solch einer Mahnung hätte „Das Jahrhundert der Politik“ vielleicht ja auch enden können. Warum eigentlich nicht?

Funding Open Access funding enabled and organized by Projekt DEAL.

Open Access Dieser Artikel wird unter der Creative Commons Namensnennung 4.0 International Lizenz veröffentlicht, welche die Nutzung, Vervielfältigung, Bearbeitung, Verbreitung und Wiedergabe in jeglichem Medium und Format erlaubt, sofern Sie den/die ursprünglichen Autor(en) und die Quelle ordnungsgemäß nennen, einen Link zur Creative Commons Lizenz beifügen und angeben, ob Änderungen vorgenommen wurden.

Die in diesem Artikel enthaltenen Bilder und sonstiges Drittmaterial unterliegen ebenfalls der genannten Creative Commons Lizenz, sofern sich aus der Abbildungslegende nichts anderes ergibt. Sofern das betreffende Material nicht unter der genannten Creative Commons Lizenz steht und die betreffende Handlung nicht nach gesetzlichen Vorschriften erlaubt ist, ist für die oben aufgeführten Weiterverwendungen des Materials die Einwilligung des jeweiligen Rechteinhabers einzuholen.

Weitere Details zur Lizenz entnehmen Sie bitte der Lizenzinformation auf http://creativecommons.org/ licenses/by/4.0/deed.de. 